\title{
Management of residual pleural space after lung resection: fully controllable paralysis of the diaphragm through continuous phrenic nerve block
}

\author{
Miriam Patella ${ }^{1}$, Andrea Saporito ${ }^{2}$, Francesco Mongelli ${ }^{1}$, Ramon Pini ${ }^{1}$, Rolf Inderbitzi ${ }^{1}$, Stefano Cafarotti ${ }^{1}$ \\ ${ }^{1}$ Department of Thoracic Surgery, ${ }^{2}$ Perioperative Medicine Research Group, San Giovanni Hospital, Bellinzona, Switzerland \\ Contributions: (I) Conception and design: S Cafarotti; (II) Administrative support: None; (III) Provision of study materials or patients: M Patella, \\ A Saporito, R Pini, R Inderbitzi, S Cafarotti; (IV) Collection and assembly of data: M Patella, F Mongelli; (V) Data analysis and interpretation: M \\ Patella, F Mongelli; (VI) Manuscript writing: All authors; (VII) Final approval of manuscript: All authors. \\ Correspondence to: Miriam Patella. San Giovanni Hospital, 6500 Bellinzona, Switzerland. Email: miriam.patella@eoc.ch.
}

\begin{abstract}
Background: Residual pleural space after lung resection associated with air leak is a challenging issue, potentially causing serious complications. We report a new, postoperative technique to reduce the pleural space, inducing a controlled and reversible paralysis of the diaphragm.

Methods: Ten patients were enrolled (7 lobectomies, 2 bilobectomy, 1 wedge resection). Inclusion criteria were: digitally detected air flow $>200 \mathrm{~mL} / \mathrm{min}$ at post-op day 3, presence of empty pleural space at chest $\mathrm{x}$-ray, absence of restrictive lung disease, absence of known arrhythmias. A $22 \mathrm{G}$ nerve-block catheter was place under ultrasound guidance in proximity to the phrenic nerve, between the sternocleidomastoid muscle and the anterior scalene muscle at the level of $6^{\text {th }}$ cervical vertebra. Continuous infusion of ropivacaine $0.2 \%$ $3 \mathrm{~mL} / \mathrm{h}$ was started. Fluoroscopy was used to confirm significant reduction in hemidiaphragm movements. Monitoring of vital signs and intense respiratory physiotherapy were enhanced. The infusion was stopped at air leak cessation and the catheter was removed along with the chest drain.

Results: No peri- and post-procedural complications occurred. In all patients, we observed an immediate reduction of the empty pleural space and resolution of the air leak within few days $(3 \pm 1.16$ days $)$. After suspension of local anaesthetic, complete restoration of the hemidiaphragm function has been documented.

Conclusions: This is an effective and minimally invasive method to reduce the residual pleural space after lung resections. Narrowing of the pleural space facilitates the contact between the lung and the chest wall promoting the resolution of the air leak. Diaphragm paralysis is controlled and temporary with no residual disabilities.
\end{abstract}

Keywords: Lung resection; residual pleural space; phrenic nerve; chest drain management

Submitted Apr 25, 2018. Accepted for publication Jun 29, 2018.

doi: $10.21037 /$ jtd.2018.07.27

View this article at: http://dx.doi.org/10.21037/jtd.2018.07.27

\section{Introduction}

Residual pleural space is a common finding after lung resection. It has been reported to occur in up to $40 \%$ of patients in the early postoperative days $(1,2)$ depending on the extent of the resection, the compliance of the remaining lung and the chest, and the underlying disease (1-3). In the vast majority of cases, this is a benign condition and its natural history leads to a complete obliteration or to a persistent space with no clinical implications (2). Usually the space is filled up with sterile fluid in first instance, and then some modifications in the physiology of the chest, such as hyperinflation of the remaining lung, shifting of the mediastinum, diaphragm elevation and narrowing of the intercostal spaces, contribute to elimination of the residual space.

Conversely, an unfavourable outcome is associated with 
Table 1 Patients characteristics

\begin{tabular}{lcccccccc}
\hline Patient & Gender & Age $>65$ & Operation & Diagnosis & BMI & PIA $>50 \%$ & FEV1 & ALPS \\
\hline 1 & M & Yes & LBiL & NSCLC & 20.6 & No & $95 \%$ & 7 \\
2 & M & Yes & LLL & NSCLC & 23.7 & No & $102 \%$ & 5 \\
3 & F & Yes & RULW & NSCLC & 18.2 & Yes & $66 \%$ & 8.5 \\
4 & M & Yes & RLL & NSCLC & 26.7 & No & $73 \%$ & 6.5 \\
5 & M & Yes & LLL & NSCLC & 20.4 & Yes & $98 \%$ & 6.5 \\
6 & F & No & LUL & Aspergilloma & 17.5 & Yes & $80 \%$ & 4 \\
7 & M & Yes & RUL & NSCLC & 32.1 & Yes & $65 \%$ & 9 \\
8 & M & Yes & UBiL & NSCLC & 24.9 & No & $95 \%$ & 8 \\
9 & M & Yes & RUL & NSCLC & 27.6 & Yes & $77 \%$ & 9 \\
10 & F & Yes & LUL & NSCLC & 21.7 & Yes & $68 \%$ & 6.5 \\
\hline
\end{tabular}

BMI, body mass index $\left(\mathrm{kg} / \mathrm{m}^{2}\right)$; PIA, pleural adhesions; FEV1, forced expiratory volume in 1 second (\%); ALPS, air leak predictor score (7); LBiL, lower bilobectomy; LLL, left lower lobectomy; RULW, right upper lobe wedge; RLL, right lower lobectomy; LUL, left upper lobectomy; RUL, right upper lobectomy; UBiL, upper bilobectomy; NSCLC, non-small cell lung cancer.

the presence of air leak and increased pleural thickness (2). The lack of contact within the parietal and visceral pleural surfaces may delay the resolution of the air leak creating a non-sterile environment and exposing the thoracic cavity to a continue source of infection. The longer this complication persist, the greater chance of serious complications, prolonging hospital stay and impacting both on health system finances and patient quality of life (4).

Plenty of studies have focused on prediction and prevention of air leak using preoperative scoring $(5,6)$ or intraoperative techniques, as many as others describing surgical interventions to treat this complication.

We report a simple method which can be used postoperatively to reduce the volume of the chest with a transient and controllable paralysis of the diaphragm, facilitating the contact between the lung and the chest wall and promoting the resolution of the air leak.

\section{Methods}

Ten patients undergoing different lung resections at our institute were enrolled between January 2016 and January 2018. Surgical characteristics and demographics are listed in Table 1. Inclusion criteria were: significant air leak $(>200 \mathrm{~mL} / \mathrm{min}$ at suction pressure of $-0.8 \mathrm{kPa}$ ) at postoperative day 3 , presence of empty pleural space at chest $\mathrm{x}$-ray either basal or apical, absence of restrictive lung disease, absence of known arrhythmias. Patients with recognized broncho-pleural fistula, lung atelectasis or pneumonia were excluded. The operations have been performed either with a three ports thoracoscopy or through an antero-lateral thoracotomy. All adhesions between the lung and the chest wall have been freed, pulmonary ligament has been divided, mechanical staplers have been used to complete the fissures, to perform the wedge resections and to close the bronchial stump in all the procedures. At the end of the lung resection, underwater test was performed in order to identify parenchymal air leaks and all attempts, including biological sealants and suturing of the lung, were made to repair them. Single $28 \mathrm{Fr}$ chest tubes were connected to a digital drain system (Thopaz, Medela, Switzerland) in all patients, with a suction pressure of $-2 \mathrm{kPa}$ postoperatively. The suction was reduced to $-0.8 \mathrm{kPa}$ if the airflow was $>800 \mathrm{~mL} / \mathrm{min}$. Chest $\mathrm{x}$-ray was performed post-operatively, when the suction pressure was reduced and at day 3 .

All patients received, as per local standard protocols, early mobilization, respiratory physiotherapy, nutritional support and pain management with regular and patientcontrolled analgesia.

A mention about the rationale behind the inclusion criteria is mandatory at this point. As there is no definitive evidence in literature about the application of suction versus no-suction after lung resection (7), we usually apply the pressure of $-2 \mathrm{kPa}$ to all patients postoperatively, with the aim of restoring the negative intra-pleural pressure and 


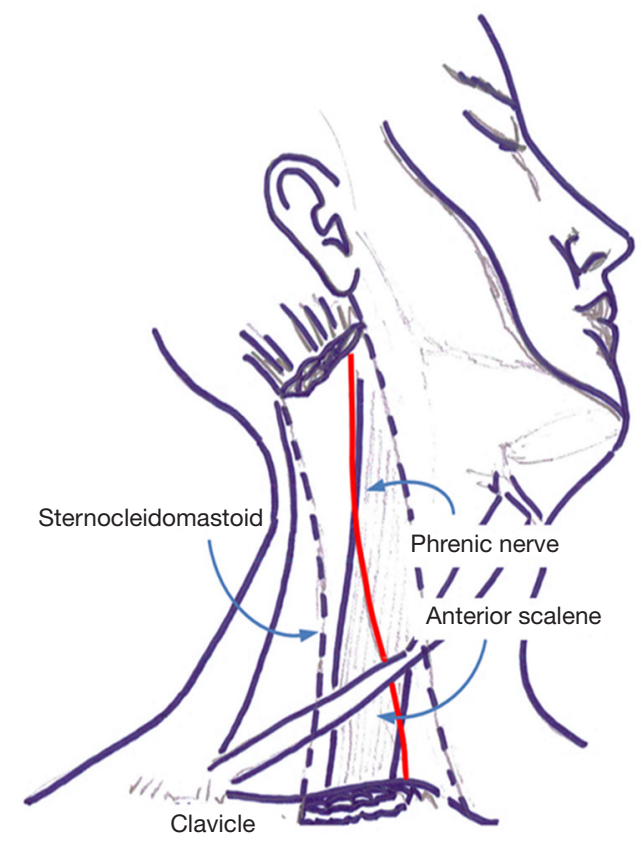

Figure 1 Anatomy of the phrenic nerve at its passage into the neck.

obtain the fullest lung expansion (7). Despite this, in all the patients in this series, residual pleural space at postoperative chest $\mathrm{x}$-ray and a very significant air leak $(>800 \mathrm{~mL} / \mathrm{min})$ were observed. With the intention to reduce the stimuli contributing to the extent or the duration of an alveolopleural fistula (7), we decreased the suction pressure to $-0.8 \mathrm{kPa}$; we actually obtained a reduction in the recorded air-leak, renouncing to obtain full lung expansion.

Thus, we considered for the phrenic nerve block those patients in whom, despite the management of the chest drain, the persistent combination of residual pleural space and air leak was likely to be prolonged.

We did not include in the study patients with restrictive lung diseases because the reduced compliance of the lung makes the diaphragm contraction greatly contributing to the respiratory function.

If the inclusion criteria were fulfilled, percutaneous ipsilateral phrenic block was proposed to the patient and informed consent was obtained by the anaesthetist performing the procedure.

Under sterile condition, the phrenic nerve was identified by ultrasound (LogiQ, GE, USA) using a $10 \mathrm{MHz}$ linear probe between the inner surface of the sternocleidomastoid muscle and the outer surface of the anterior scale muscle at

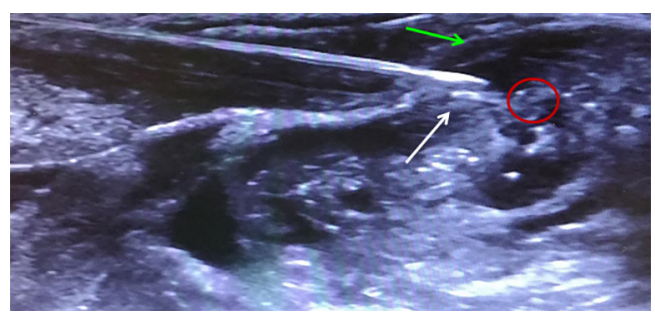

Figure 2 Ultrasound shows the needle reaching the phrenic nerve (circled), between the anterior scalene muscle (white arrow) and the sternocleidomastoid muscle (green arrow).

the level of $6^{\text {th }}$ cervical vertebra (Figure 1).

A continuous anesthetic block of the phrenic nerve was performed using a $22 \mathrm{G}$ nerve-block catheter (Contiplex $\mathrm{C}, \mathrm{BBraun}, \mathrm{Germany})$, inserted in-plane under real-time ultrasound guidance using a catheter-over-the-needle technique with a lateral-to-medial technique.

Needle tip position was considered optimal when placed in the plane between the fasciae of the sternocleidomastoid and the anterior scalene muscles, as confirmed by the administration of a bolus of $1 \mathrm{~mL}$ normal saline under direct ultrasound guidance, at about $0.5 \mathrm{~cm}$ from the phrenic nerve (Figure 2). A first anesthetic bolus of $3 \mathrm{~mL}$ ropivacaine $0.75 \%$ was than administered through the needle before this was retracted, leaving the catheter in place. The local anesthetic spread pattern was observed to make sure it reached the phrenic nerve. The perineural catheter was fixed using Sterile Strips and a transparent drape and, after a negative aspiration test, a continuous infusion of ropivacaine $0.2 \% 3 \mathrm{~mL} / \mathrm{h}$ was started, via a disposable elastomeric pump (Easypump, BBraun, Germany) with an adjustable infusion rate. The technique is described in Figure 3. Fluoroscopy was used to confirm significant reduction in hemidiaphragm movements. Closer monitoring of vital signs and intense respiratory physiotherapy were enhanced, and all patients had continuous recording of air-leak with digital drain system. The infusion was stopped at air leak cessation and the catheter was removed along with the chest drain. After 4 hours from the drain removal, a chest $x$-ray at the end of full inspiration was performed in order to assess the absence of complication and the restoration of the diaphragm function.

The project was evaluated by the local Ethics Committee (Comitato Etico Cantonale, Ticino, Switzerland) which stated that it does not need an approval since the data were fully anonymized. 


\section{Results}

Ten patients fulfilled the inclusion criteria within a period of 2 years. Nine had a diagnosis of primary lung cancer while 1 had an aspergilloma of the left upper lobe. The 9 anatomical lung resections included 1 lower bilobectomy, 1 upper bilobectomy, 3 lower lobectomies ( 2 left and 1 right) and 4 upper lobectomies ( 2 left and 2 right). The

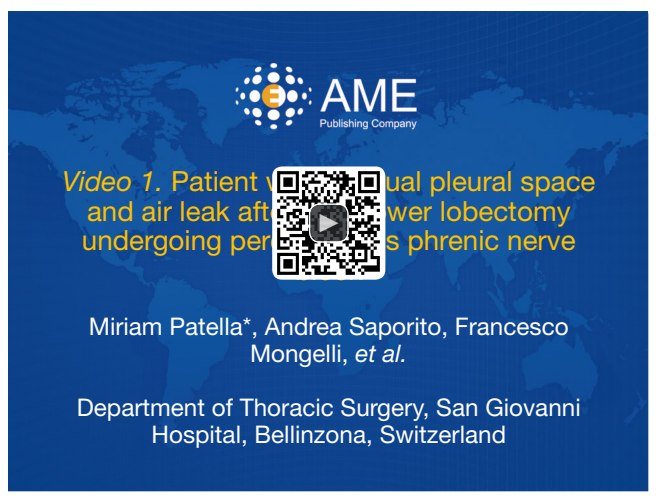

Figure 3 Patient with residual pleural space and air leak after right lower lobectomy undergoing percutaneous phrenic nerve block (8). Before the procedure, the registered airflow at digital drain system was $200-400 \mathrm{~mL} / \mathrm{min}$. The air leak resolved within two days after the nerve block with complete restoration of diaphragm function after discontinuation of ropivacaine infusion.

Available online: http://www.asvide.com/article/view/26625 remaining wedge resection was performed in a patient with previous right lower lobectomy who developed a second adenocarcinoma of the lung in the right upper lobe.

After the phrenic nerve block, we observed a significant reduction in the ipsilateral diaphragm motion and an immediate reduction of the empty pleural space in all patients (Figure 4).

There were no peri- o post-procedural complications: nor respiratory failure or distress neither arrhythmias have been documented. The management of the elastomeric pump resulted immediate for the nursing staff and it did not cause any restriction to the mobilization of the patient. Conversely, patients have been encouraged to mobilize actively with the physiotherapists to avoid sputum retention and to help the lung expansion. The resolution of the air leak was obtained in $3 \pm 1.16$ days on average. At the chest $\mathrm{x}$-ray performed after the removal of the catheter and the chest drain, we observed the complete obliteration of the residual space in 8 patients and the persistence of a small asymptomatic space in 2 . The film obtained at the end of full inspiration demonstrated the normal contraction of the diaphragm. All patients have been followed-up in outpatient clinic within 2 weeks and there have been no re-admissions related to the surgery.

\section{Discussion}

The association of residual pleural space and air leak
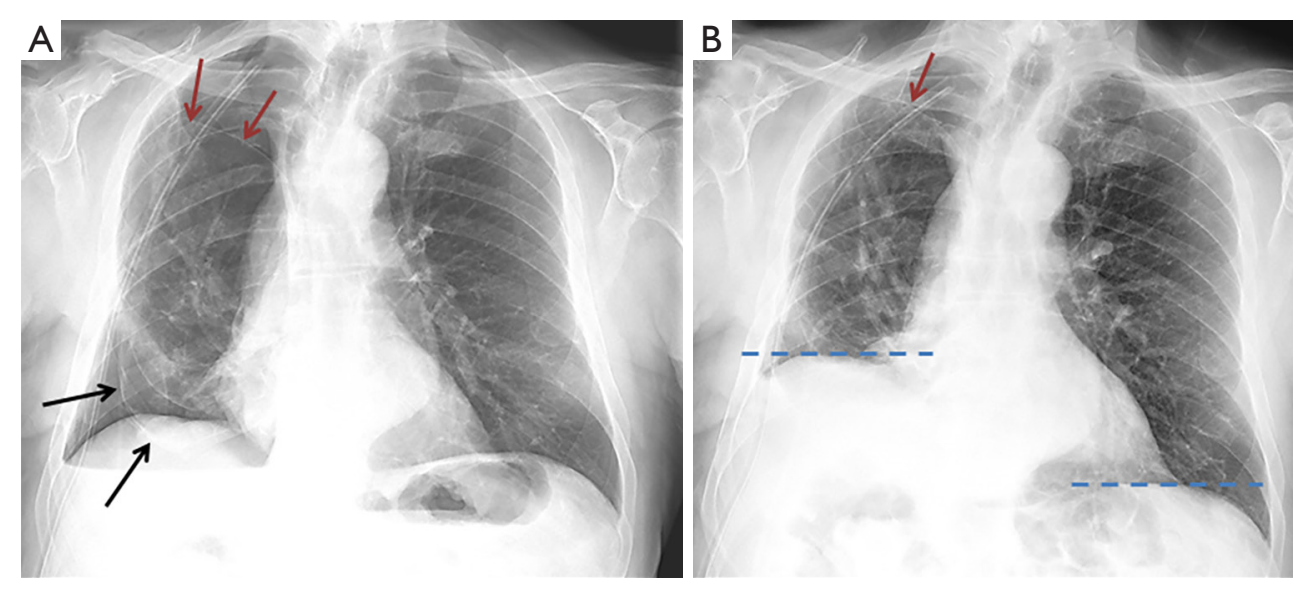

Figure 4 Radiological findings before and after phrenic nerve block. (A) Patient with right lower lobectomy, persistent air leak, basal (black arrows) and apical (red arrows) space; (B) the same patient after percutaneous phrenic block. Full inspiration chest film shows paralysis of right hemidiaphragm compared with the counterpart (dotted lines), the obliteration of the basal space and the consistent reduction of the apical space (red arrow). 
after lung resection may represent a source of serious complications. This condition is relatively common when dealing with conditions reducing the compliance of the chest or when a large parenchymal resection is needed. Concurrent or previous inflammatory or infectious disease, previous thoracic intervention, radiotherapy or resection performed in emphysematous or fibrotic lung increase the risk of post-residual space. The presence of alveolar air leak which is likely to be prolonged, delays the chest drain removal, exposes patients to high risk of infection and influences the length of stay in hospital (9-11).

Several treatments have been described to fill the residual space and promoting resolution of air leak, such as transposition of muscle flaps or omentum or even thoracoplasty: they are invasive and sometimes disabling. We have to consider that prolonged air leak affects more often frail patients (5) and the indications for initial surgical resection and the diseases for which it was required often are sufficiently debilitating; a surgical re-intervention can be potentially more harmful than the air-leak itself.

The treatment of choice must imply a global consideration of the risks and the benefits of the procedure per single patient, taking into account performance status, respiratory and systemic disease and prognosis, problems with maintaining the drainage, infection, as well as social and even economic aspects (3).

Decision-making may not always be easy, and less invasive procedures have been proposed overtime.

The first conservative approach includes prolonged chest tube drainage. It is still debated if the application of suction at the chest drainage system could worsen the alveolar leak or just speed up the expansion of the lung and favour the contact with the chest wall (12-15). However, the hyper-expansion of the residual lung that has to fill up a pleural cavity that previously hosted a larger lung, results in overdistention of the alveoli (4): there are good experimental works supporting the notion that lung overdistension favours air leak and furthermore represents an edemagenic condition (16). Hence, surgeons must "resist the temptation" of applying high suction to attract the lung towards the chest wall, especially in case of reduced chest compliance. It is apparent that patients can live in relatively good health for long periods of time with persisting vented spaces in association with air leak from alveolar seepage sources, even with the presence of welldrained and controlled empyema (3). The implementation of home nursing services promoted the use of Heimlich valve in order to discharge patients from hospital with the chest drain still in situ. However, severe complications are rare but possible $(17,18)$ and not many patients will agree with ambulatory management.

To avoid this challenging issue, many authors have proposed intraoperative techniques in order to immediately obliterate the pleural cavity and to prevent prolonged air leak. Pleural tenting, pneumoperitoneum and crushing of the phrenic nerve are the most commonly used. However, all of them present various shortfalls: pleural tenting may not be technically feasible (19); pneumoperitoneum can be performed either intraoperatively (20) or postoperatively (21), but requires access to the abdomen and once instituted it is not immediately reversible if needed; crushing of the phrenic nerve can lead to a permanent dysfunction of the muscle (22).

Other techniques to reduce the rate of post-resection residual space include cryoneuroablation of phrenic nerve (23), and injection of Botulinum toxin type A has been studied in animal models (24). Both these strategies produce temporary and fully reversible paralysis of the ipsilateral diaphragm, but the effect is not controllable at any time and it lasts 30 to 60 days.

More interesting appears to be the use of local anaesthetic, continuously delivered by a catheter placed under direct view, during video assisted surgery or thoracotomy, when a space problem is likely to occur $(25,26)$. In both studies, the authors described the use of an epidural catheter, secured in proximity to the phrenic nerve in its intrathoracic course, which releases lidocaine $1 \%$ at $3 \mathrm{~mL} / \mathrm{h}$ and bupivacaine $0.25 \%$ at $5 \mathrm{~mL} / \mathrm{h}$ respectively. They obtained substantial reduction in chest volume, good results in the management of the air leak and complete restoration of diaphragm function after discontinuation of the infusion. These preliminary experiences are encouraging with the only pitfall of the need of inserting the catheter during the surgical procedure.

The technique we described combines the manageability of the local anaesthetic with the option of establish the infusion whenever needed with a minimally invasive approach.

Unilateral diaphragmatic paralysis is described as been a common complication during interscalene brachial plexus block with local anaesthetic for upper limb surgery (27). The effect usually lasts few hours after the injection and it resolves completely afterwards (28). We hypothesized to use this effect, specifically targeting the phrenic nerve, to temporary paralyse the hemidiaphragm in case of postresection residual space and parenchymal air leak. 
Raising the diaphragm ultimately results in a reduction of the chest volume promoting the symphysis of two pleural surfaces and the sealing of small air leaks from the lung, as postulated by Rice et al. (29). Once the air leak has stopped, the presence of a persistent pleural space after the removal of chest drain does not compromise the outcome. Two of our patients had a persistent space after removal of the drain. Both of them had a lower lobectomy, with a probable source of air leak from the lower surface of the lung which was manipulated most, while the residual space remained apically. This does not impair the promotion of healing process achieved with pleural apposition.

The advantages of this technique compared to the previous described are various: it is minimally invasive, fully controllable and immediately reversible. The use of ultrasound guidance ensures correct and safe placement of the catheter. The chance of begin it postoperatively allows a good candidates selection and avoids unnecessary procedures and exposure to side effects and complications.

Even though in our small series of patients we did not observe any complications, the use of bupivacaine is rarely associated with seizures, with no impact on the cardiovascular system (30) and in a review of the literature on extra-pleural bupivacaine infusion, local complications occurred in $0.6 \%$ of patients and systemic complications in $0.8 \%$ (31). Moreover, direct effects of diaphragm paralysis may be respiratory impairment and sputum retention. To avoid, or eventually early recognize these issues, we implemented close vital signs monitoring and we enhanced the routine respiratory physiotherapy. Suspension of the infusion allows prompt restoration of muscle's function and recovery from hypoventilation.

Looking at the patients' demographics, we can make a retrospective consideration: the literature reports some factors related to patients characteristics and kind of surgery which predict the risk of developing prolonged air leak postoperatively. This aggregate score, specifically designed for lobectomies, assigns an odd risk to each patient based on the most common predictors for prolonged air leak including age $>65$ years, forced expiratory volume in 1 second (FEV1) $<80 \%$, male sex, pleural adhesions, Body Mass Index $<25.5 \mathrm{~kg} / \mathrm{m}^{2}$, upper lobes and right side of lung resection (7). This score system, which needs validation in forthcoming studies, helps the management of chest tubes and suction. If we speculate on our ten patients, we can easily appreciate that they encounter most risk factors, therefore, a similar model could be used to better define the indications for the use of percutaneous phrenic block.
Moreover, a time-frame consideration needs to be made: if the combination of residual space and consistent air leak is present post-operatively and the risk of prolonged air leak is high, is probably advisable to early consider the phrenic block, before pleuro-parenchymal adhesions occur. This could maximise the effect of diaphragm elevation also for apical spaces. On the other hand, air leak cut-off and timing of nerve block were chosen arbitrary: post-operative day 3 is a relatively short time to propose an intervention, but, having considered the minimal invasive technique of the procedure and the patients' risk-factors associated with prolonged air leak, this seems reasonable.

The present study describes a new technique with preliminary good results, however, the number of the included patients is small, and definitive conclusions on feasibility and improvement of the outcome cannot be expressed.

In conclusion, transcutaneous phrenic block appears to be a safe and effective technique to reduce the postresection residual space. Further studies needs to be conducted on larger series to better assess possible issues related to the procedure, standardize target population and timing.

\section{Acknowledgements}

None.

\section{Footnote}

Conflicts of Interest: The authors have no conflicts of interest to declare.

Ethical Statement: The project was evaluated by the local Ethics Committee (Comitato Etico Cantonale, Ticino, Switzerland) which stated that it does not need an approval since the data were fully anonymized.

\section{References}

1. Solak O, Sayar A, Metin M, et al. Definition of postresectional residual pleural space. Can J Surg 2007;50:39-42.

2. Misthos P, Kokotsakis J, Konstantinou M, et al. Postoperative residual pleural spaces: characteristics and natural history. Asian Cardiovasc Thorac Ann 2007;15:54-8.

3. Barker WL. Natural history of residual air spaces 
after pulmonary resection. Chest Surg Clin N Am 1996;6:585-613.

4. Pompili C, Miserocchi G. Air leak after lung resection: pathophysiology and patients' implications. J Thorac Dis 2016;8:S46-54.

5. Brunelli A, Varela G, Refai $M$ et al. A scoring system to predict the risk of prolonged air leak after lobectomy. Ann Thorac Surg 2010;90:204-9.

6. Lee L, Hanley SC, Robineau C, et al. Estimating the risk of prolonged air leak after pulmonary resection using a simple scoring system. J Am Coll Surg 2011;212:1027-32.

7. Rocco G, Brunelli A, Rocco R. Suction or Nonsuction: How to Manage a Chest Tube After Pulmonary Resection. Thorac Surg Clin 2017;27:35-40.

8. Patella M, Saporito A, Mongelli F, et al. Patient with residual pleural space and air leak after right lower lobectomy undergoing percutaneous phrenic nerve block. Asvide 2018;5:708. Available online: http://www.asvide. com/article/view/26625

9. Stéphan F, Boucheseiche S, Hollande J, et al. Pulmonary complications following lung resection: a comprehensive analysis of incidence and possible risk factors. Chest 2000;118:1263-70.

10. Brunelli A, Xiume F, Al Refai M, et al. Air leaks after lobectomy increase the risk of empyema but not of cardiopulmonary complications: a case-matched analysis. Chest 2006;130:1150-6.

11. Varela G, Jiménez MF, Novoa N, et al. Estimating hospital costs attributable to prolonged air leak in pulmonary lobectomy. Eur J Cardiothorac Surg 2005;27:329-33.

12. Alphonso N, Tan C, Utley M, et al. A prospective randomized controlled trial of suction versus non-suction to the under-water seal drains following lung resection. Eur J Cardiothorac Surg 2005;27:391-4.

13. Brunelli A, Monteverde M, Borri A, et al. Comparison of water seal and suction after pulmonary lobectomy: a prospective, randomized trial. Ann Thorac Surg 2004;77:1932-7; discussion 1937.

14. Cerfolio RJ, Bass C, Katholi CR. Prospective randomized trial compares suction versus water seal for air leaks. Ann Thorac Surg 2001;71:1613-7.

15. Marshall MB, Deeb ME, Bleier JI, et al. Suction vs water seal after pulmonary resection: a randomized prospective study. Chest 2002;121:831-5.

16. Miserocchi G, Negrini D, Gonano C. Parenchymal stress affects interstitial and pleural pressures in in situ lung. $\mathrm{J}$ Appl Physiol (1985) 1991;71:1967-72.

17. Tavare AN, Creer DD, Khan S, et al. Ambulatory percutaneous lung biopsy with early discharge and Heimlich valve management of iatrogenic pneumothorax: more for less. Thorax 2016;71:190-2.

18. Schmocker RK, Vanness DJ, Macke RA, et al. Outpatient air leak management after lobectomy: a CMS cost analysis. J Surg Res 2016;203:390-7.

19. Allama AM. Pleural tent for decreasing air leak following upper lobectomy: a prospective randomised trial. Eur J Cardiothorac Surg 2010;38:674-8.

20. Toker A, Dilege S, Tanju S, et al. Perioperative pneumoperitoneum after lobectomy -- bilobectomy operations for lung cancer: a prospective study. Thorac Cardiovasc Surg 2003;51:93-6.

21. De Giacomo T, Rendina EA, Venuta F, et al. Pneumoperitoneum for the management of pleural air space problems associated with major pulmonary resections. Ann Thorac Surg 2001;72:1716-9.

22. Iverson LI, Mittal A, Dugan DJ, et al. Injuries to the phrenic nerve resulting in diaphragmatic paralysis with special reference to stretch trauma. Am J Surg 1976;132:263-9.

23. Pan XJ, Ou DB, Lin X, et al. Management of Pleural Space After Lung Resection by Cryoneuroablation of Phrenic Nerve: A Randomized Study. Surg Innov 2017;24:240-4.

24. Kaya SO, Atalay H, Erbay HR, et al. Exploring strategies to prevent post-lobectomy space: transient diaphragmatic paralysis using Botulinum toxin type A (BTX-A). Int Semin Surg Oncol 2005;2:21.

25. Carboni GL, Vogt A, Küster JR, et al. Reduction of airspace after lung resection through controlled paralysis of the diaphragm. Eur J Cardiothorac Surg 2008;33:272-5.

26. Clavero JM, Cheyre JE, Solovera ME, et al. Transient diaphragmatic paralysis by continuous para-phrenic infusion of bupivacaine: a novel technique for the management of residual spaces. Ann Thorac Surg 2007;83:1216-8.

27. Stundner O, Meissnitzer M, Brummett CM, et al. Comparison of tissue distribution, phrenic nerve involvement, and epidural spread in standard- vs lowvolume ultrasound-guided interscalene plexus block using contrast magnetic resonance imaging: a randomized, controlled trial. Br J Anaesth 2016;116:405-12.

28. Ip VH, Tsui BC. Continuous interscalene block: the good, the bad and the refined spread. Acta Anaesthesiol Scand 2012;56:526-30.

29. Rice TW, Okereke IC, Blackstone EH. Persistent air-leak following pulmonary resection. Chest Surg Clin N Am 2002;12:529-39. 
30. Brown DL, Ransom DM, Hall JA, et al. Regional anesthesia and local anesthetic-induced systemic toxicity: seizure frequency and accompanying cardiovascular changes. Anesth Analg 1995;81:321-8.

Cite this article as: Patella M, Saporito A, Mongelli F, Pini R, Inderbitzi R, Cafarotti S. Management of residual pleural space after lung resection: fully controllable paralysis of the diaphragm through continuous phrenic nerve block. J Thorac Dis 2018;10(8):4883-4890. doi: 10.21037/jtd.2018.07.27
31. Detterbeck FC. Efficacy of methods of intercostal nerve blockade for pain relief after thoracotomy. Ann Thorac Surg 2005;80:1550-9. 